\title{
Environmental enrichment mitigates the long-lasting sequelae of perinatal fentanyl exposure
}

\author{
Jason Bondoc Alipio ${ }^{1 *}$, Lace Marie Riggs ${ }^{2 *}$, Madeline Plank $^{1}$, Asaf Keller ${ }^{1}$
}

${ }^{1}$ Department of Anatomy \& Neurobiology, Program in Neuroscience, University of Maryland School of Medicine, Baltimore, MD 21201

${ }^{2}$ Department of Psychiatry, Division of Translational and Basic Science, Program in

Neuroscience and Training Program in Integrative Membrane Biology, University of Maryland School of Medicine, Baltimore, MD 21201

* Authors contributed equally to this study

Corresponding Author: Asaf Keller, Department of Anatomy \& Neurobiology, University of Maryland School of Medicine, 20 Penn Street, Baltimore, MD 21201, akeller@som.umaryland.edu 


\begin{abstract}
The opioid epidemic is a rapidly evolving societal issue driven, in part, by a surge in synthetic opioid use. A rise in fentanyl use among pregnant women has led to a 40-fold increase in the number of perinatally-exposed infants in the past decade. These children are more likely to develop mood- and somatosensory-related conditions later in life, suggesting that fentanyl may permanently alter neural development. Here, we examined the behavioral and synaptic consequences of perinatal fentanyl exposure in adolescent male and female C57BL/6J mice and assessed the therapeutic potential of environmental enrichment to mitigate these effects. Dams were given ad libitum access to fentanyl $(10 \mu \mathrm{g} / \mathrm{mL}$, per os $)$ across pregnancy and until weaning (PD 21). Perinatally-exposed adolescent mice displayed hyperactivity (PD 45), enhanced sensitivity to anxiogenic environments (PD 46), and sensory maladaptation (PD 47) - sustained behavioral effects that were completely normalized by environmental enrichment (PD 21-45). Additionally, environmental enrichment normalized the fentanyl-induced changes in the frequency of miniature excitatory postsynaptic currents of layer $2 / 3$ neurons in the primary somatosensory cortex (S1). We also demonstrate that fentanyl impairs short- and long-term potentiation in S1 layer 2/3 neurons which, instead, exhibit a sustained depression of synaptic transmission that is restored by environmental enrichment. On its own, environmental enrichment suppressed long-term depression of control S1 neurons from vehicle-treated mice subjected to standard housing conditions. These results demonstrate that the lasting effects of fentanyl can be ameliorated with a non-invasive intervention introduced during early development.
\end{abstract}

Keywords: Perinatal opioid exposure; fentanyl; environmental enrichment; plasticity; somatosensory cortex; anxiety; attention deficit hyperactivity disorder; adolescence; intervention

\title{
Significance Statement
}

Illicit use of fentanyl accounts for a large proportion of opioid-related overdose deaths. Children exposed to opioids during development have a higher risk of developing neuropsychiatric disorders later in life. Here, we employ a preclinical model of perinatal fentanyl exposure that recapitulates these long-term impairments and show, for the first time, that environmental enrichment can reverse deficits in somatosensory circuit function and behavior. These findings have the potential to directly inform and guide ongoing efforts to mitigate the consequences of perinatal opioid exposure. 


\section{Introduction}

The prevalence of opioid use disorder has steadily increased since the early 1990s, with women representing more than one-third of opioid users in the United States (Degenhardt et al., 2018). While opioid overdose deaths were initially driven by misuse of prescription opioids (like morphine) and their illicit counterparts (e.g., heroin), synthetic opioids, such as fentanyl, are now the main catalyst of overdose deaths (O'Donnell et al., 2017; Jannetto et al., 2019). Just among women 30-34 years of age, the rate of these overdose deaths have increased 35-fold in the last two decades (from 0.31 to 11 deaths per 100,000; VanHouten et al., 2019). Opioid use by pregnant women is a growing public health concern, with a $400 \%$ increase in the number of infants born to opioid-using mothers (from 1.5 to 6.5 per 1000 infants born; Haight et al., 2018). Opioid use during pregnancy increases the risk of miscarriage, premature birth, and stillbirth (Whiteman et al., 2014) whereas the infants who are born suffer from neonatal opioid withdrawal syndrome (NOWS; Winkelman et al., 2018). Often, NOWS is met with morphine, methadone, and buprenorphine treatment immediately after birth (Sutter et al., 2014), likely further compounding the developmental consequences of perinatal opioid exposure itself.

Longitudinal clinical studies indicate an increased risk of developing psychiatric conditions among children with a history of in utero and iatrogenic opioid exposure (Sherman et al., 2019). Specifically, these children have a higher incidence of anxiety (Cubas and Field, 1993), attention-deficit hyperactivity (Ornoy et al., 2001; Nygaard et al., 2016; Schwartz et al., 2021), autism spectrum (Sherman et al., 2019), and sensory processing disorders (Kivistö et al., 2014). Impairments in sensory processing is of particular interest because it is a common symptom in syndromes like attention-deficit hyperactivity and autism spectrum disorders (Ayres, 1964; Robertson and Baron-Cohen, 2017). While infants suffering from NOWS typically receive treatment for the acute symptoms of opioid withdrawal, there is virtually no treatment approaches specifically designed to address the other neurodevelopmental consequences that these children face across their lifetime.

Environmental enrichment in rodents can reverse early neurodevelopmental insults (Nithianantharajah and Hannan, 2006) and improve cognitive (Rijzingen et al., 1997; Passineau et al., 2001) and emotional functioning later in life (Brenes et al., 2009). This is due in part to the restoration of synaptic plasticity (Bayat et al., 2015) and neurogenesis (Gaulke et al., 2005), which are believed to re-establish neuronal complexity (Wei et al., 2021) via brain-derived neurotrophic factor signaling (Chen et al., 2005). While the synaptic benefits of environmental enrichment are often ascribed to its actions within corticolimbic circuitry, some of the most fundamental studies to establish its importance to synaptic efficacy took place within the somatosensory cortex (Smail et al., 2020). We previously showed that perinatal fentanyl exposure increases anxiety-like behavior and impairs synaptic transmission in anterior cingulateand primary somatosensory (S1) cortex (Alipio et al., 2021a, 2021b). Because environmental enrichment can reduce anxiety-like behavior and restore the functional integrity of cortical circuits (Brenes et al., 2009; Wei et al., 2021), we hypothesized that environmental enrichment would mitigate the behavioral and synaptic deficits of perinatal fentanyl exposure.

\section{Materials and Methods}

Animals. All procedures were reviewed and approved by the University of Maryland Institutional Animal Care and Use Committee and adhered to the National Institutes of Health guide for the care and use of laboratory animals and Animal Research Reporting of In Vivo Experiments 
guidelines. Male and female C57BL/6J mice were used and bred in our temperature and humidity-controlled vivarium. When copulatory plugs were identified, we removed the sires and added fentanyl $(10 \mu \mathrm{g} / \mathrm{mL}$ in $2 \%$ saccharin) or vehicle control (2\% saccharin) to the water hydration pouches for ad libitum access by dams. Offspring were weaned at postnatal day (PD) 21 and housed 2-5 mice/cage/sex in standard housing, or 6-8 mice/cage/sex in enriched housing. Enriched housing was custom made from two One Cage $2100^{\mathrm{TM}}$ Micro-Isolator systems (Lab Products LLC, Seaford, DE). Enriched housing had a floor area of $420 \mathrm{in}^{2}$ and included a variety of solid items for the mice to interact with (Fig 1. and Table 1). Food and water were available ad libitum and lights were maintained on a 12-hour cycle (0700 lights-on). Mice were tested in the open field test on PD 45 and in the tactile withdrawal test on PD 47. Slice experiments took place from PD 48-55.

Statistical Analyses. We adhered to accepted standards for rigorous study design and reporting to maximize the reproducibility and translational potential of our findings as described in Landis et al. (Landis et al., 2012) and in ARRIVE Guidelines (Kilkenny et al., 2010). Dams were randomly allocated to receive fentanyl or vehicle. In all our experiments, the primary end of points were prospectively defined for each experiment. All experimenters were blind to treatment conditions throughout data collection, scoring, and analysis. Statistical analyses were conducted using Prism v9 (GraphPad, San Diego, CA) and the minimum sample size was determined using G*Power Software Suite (Heinrich-Heine, Universität Düsseldorf). Statistical significance was defined as $p<0.05$. No significant sex differences were observed for any of the experimental outcomes, thus, data from male and female mice were combined. There were no differences between mice of different litters within the same drug exposure group, therefore, individual mice served as a single sample count. For electrophysiology data, all neurons from a single mouse were averaged and served as a single sample count (Editorial, 2018). Student's $t$ tests were used for two-group comparisons and the effect size was determined using Cohen's $d$ and defined as small $(\leq 0.2)$, medium $(\leq 0.5)$, or large $(\geq 0.8)$. One-way repeated measures analysis of variance (AVOVA) was used when housing condition (standard vs. enriched) and time (repeated-measure) were independent variables. Two-way ANOVA was used when drug condition (vehicle vs. fentanyl) and housing condition were independent variables. Three-way repeated-measures ANOVA was used when drug condition, housing condition, and time were independent variables. When a significant main effect and/or interaction was observed, Tukey or Bonferroni post-hoc tests were used to assess pairwise comparisons and partial eta squared $\left(\mathrm{P}^{2}\right)$ was used to determine if the effect size of the comparison was small $(\leq 0.01)$, medium $(\leq 0.06)$, or large ( $\geq 0.14)$. Fisher's exact tests were used for contingency occurrence of plasticity (LTP vs. no LTP; LTD vs. no LTD) and odds ratio was used to determine if the effect size was small $(\leq$ $1.49)$, medium $(\leq 3.45)$, or large $(\geq 9)$. Kruskal-Wallis tests were used for cumulative probability plots and $\mathrm{P \eta}^{2}$ was used to determine effect size of those comparisons. Nonparametric alternatives were used if the data did not pass Spearman's test for heteroscedasticity and D'Agostino-Pearson omnibus K2 test of distribution normality. For two-group nonparametric comparisons, Glass' delta or Hedges' $g$ were used to determine if the effect size was small $(\leq 0.2)$, medium $(\leq 0.5)$, or large $(\geq 0.8)$ whereas $\mathrm{P \eta}^{2}$ was used to determine the effect size when three or more groups were being compared.

Open Field Test was used to assess general locomotor activity and anxiety-like behavior. Mice habituated to the testing room for at least 1-hr before the testing session began. Mice were individually placed in an open field arena $(49 \times 49 \times 49 \mathrm{~cm}$; San Diego Instruments, San Diego, 
CA) along the outside edge, facing the wall, and were allowed to freely explore the chamber for 30-min. The testing room was dimly lit with warm incandescent floor lamps and the center floor of the chamber read $\sim 30$ lux. The test was recorded by an overhead digital camera, and distance traveled $(\mathrm{cm})$ and time spent in the center (defined as $50 \%$ of the center area) were automatically scored using TopScan Software (CleverSys Inc, Reston, VA). Reduced time spent in the center zone of the arena is considered an anxiety-like response in this task.

Tactile Hind Paw Withdrawal Test was used to assess sensory threshold and adaptation. Mice habituated to the testing room for at least 1-hr before the testing session began, and were habituated to an elevated clear plexiglass box with a mesh bottom for $10 \mathrm{~min}$. We applied von Frey filaments of increasing forces (in grams: 0.16, 0.40, 0.60, 1.00, 1.40, 2.00) to the plantar surface of the hind paw. A response was defined as an active withdrawal of the paw from the probing filament. The filament was applied to the same paw throughout the test. We used the updown method to determine withdrawal threshold, as previously described (Dixon, 1965; Chaplan et al., 1994; Deuis et al., 2017). To assess sensory adaptation, we applied a von Frey filament one step above threshold to the plantar surface of the hind paw, opposite to the hind paw used during tactile sensitivity testing. The filament was applied once every $30 \mathrm{~s}$ until the animal stopped responding. The number of times the animals responded was counted, with persistent responding to tactile stimulation indicating sensory maladaptation

Drugs and Solutions. Dams' water hydration pouches contained either $10 \mu \mathrm{g} / \mathrm{mL}$ fentanyl citrate (calculated as free base) in $2 \%(\mathrm{w} / \mathrm{v})$ saccharin or $2 \%$ saccharin (vehicle control), replenished weekly until litters were weaned on PD 21. Artificial cerebrospinal fluid (ACSF) compositions and slice preparations were based on slice collection methods of Ting et al. (Ting et al., 2014) $\mathrm{N}$ methyl-D-glucamine (NMDG) ACSF contained (in mM): $92 \mathrm{NMDG}, 30 \mathrm{NaHCO}_{3}, 20 \mathrm{HEPES}$, 25 glucose, $5 \mathrm{Na}$-ascorbate, 2 thiourea, $1.25 \mathrm{NaH}_{2} \mathrm{PO}_{4}, 2.5 \mathrm{KCl}, 3 \mathrm{Na}$-pyruvate, $0.5 \mathrm{CaCl}_{2} \cdot 2 \mathrm{H}_{2} \mathrm{O}$ and $10 \mathrm{MgSO}_{4} \cdot 7 \mathrm{H}_{2} \mathrm{O}$. Holding ACSF contained (in mM): $120 \mathrm{NaCl}, 2.5 \mathrm{KCl}, 1.25 \mathrm{NaH}_{2} \mathrm{PO}_{4}, 24$ $\mathrm{NaHCO}_{3}, 12.5$ glucose, $2 \mathrm{MgSO}_{4} \cdot 7 \mathrm{H}_{2} \mathrm{O}$, and $2 \mathrm{CaCl}_{2} \cdot 2 \mathrm{H}_{2} \mathrm{O}$. Recording ACSF contained (in $\mathrm{mM}$ ): $120 \mathrm{NaCl}, 3 \mathrm{KCl}, 1 \mathrm{NaH}_{2} \mathrm{PO}_{4}, 25 \mathrm{NaHCO}_{3}, 20$ glucose, $1.5 \mathrm{MgSO}_{4} \cdot 7 \mathrm{H}_{2} \mathrm{O}$, and 2.5 $\mathrm{CaCl}_{2} \cdot 2 \mathrm{H}_{2} \mathrm{O}$. ACSF pH was adjusted to 7.4 and osmolarity to $305 \pm 2 \mathrm{mOSm}$. All ACSF solutions were saturated with carbogen $\left(95 \% \mathrm{O}_{2}\right.$ and $\left.95 \% \mathrm{CO}_{2}\right)$. Patch pipettes contained (in $\mathrm{mM}$ ): 130 cesium methanesulfonate, 10 HEPES, 1 magnesium chloride, 2.5 ATP-Mg, 0.5 EGTA, 0.2 GTP-Tris, 5 QX-314, and 2\% biocytin. The $\mathrm{pH}$ of the internal pipette solution was adjusted to 7.3 and osmolarity to $290 \pm 2 \mathrm{mOSm}$. To isolate excitatory postsynaptic currents (EPSCs), gabazine $(1 \mu \mathrm{M})$ was included in the ACSF. For miniature EPSC (mEPSC) recordings, tetrodotoxin $(1 \mu \mathrm{M})$ was included in the ACSF. All recordings were obtained at room temperature.

Slice Preparation. Mice were deeply anesthetized with intraperitoneal injection of ketamine (180 $\mathrm{mg} / \mathrm{kg})$ and xylazine $\left(20 \mathrm{mg} / \mathrm{kg}\right.$ ) then transcardially perfused with ice-cold $\left(4^{\circ} \mathrm{C}\right)$ NMDG ACSF. Their brains were rapidly extracted following decapitation. Coronal slices $(300 \mu \mathrm{m}$ thick) containing the primary somatosensory cortex (S1) were cut in ice-cold $\left(4^{\circ} \mathrm{C}\right)$ NMDG ACSF using a Leica VT1200s vibratome (Leica Biosystems, Buffalo Grove, IL) and transferred to warm $\left(33^{\circ} \mathrm{C}\right) \mathrm{NMDG}$ ACSF for $10 \mathrm{~min}$. The slices were then transferred to warm $\left(33^{\circ} \mathrm{C}\right)$ holding ACSF and allowed to cool to room temperature $\left(20-22^{\circ} \mathrm{C}\right)$ for at least $45 \mathrm{~min}$ before electrophysiology recordings. 
Electrophysiology. Whole cell patch-clamp recordings were obtained from S1 layer 2/3 neurons with a Multiclamp 700B amplifier (Molecular Devices, San Jose, CA) low-pass filtered at 1.8 $\mathrm{kHz}$ with a four-pole Bessel filter, and digitized with Digidata 1440A (Molecular Devices). Slices were placed in a submersion chamber and continually perfused $(>2 \mathrm{~mL} / \mathrm{min})$ with recording ACSF. Neurons were visually identified by infrared differential interference contrast imaging and location and neuronal morphology verified after each recording with biocytin immunohistochemistry. Borosilicate patch pipettes had an impedance of 4-6 M $\Omega$. Once G $\Omega$ seal was obtained, neurons were held in voltage-clamp configuration at $-70 \mathrm{mV}$ and the input resistance, resting membrane potential, and capacitance were measured. Series resistance $(<30$ $\mathrm{M} \Omega$ ) was monitored throughout recordings and recordings were discarded if series resistance changed by $>20 \%$ from baseline. Concentric bipolar tungsten electrodes were used to deliver electrical stimulation ( $0.2 \mathrm{~ms}$ duration) in S1 layer 5, below the recorded neuron. Electricallyevoked current responses were recorded at 1.5-fold threshold, defined as the minimum stimulation intensity required to produce a visible current response beyond baseline noise.

Long-term potentiation (LTP) was induced using a pairing induction protocol: 80 stimulation pulses at $2 \mathrm{~Hz}$ paired with postsynaptic depolarization to $+30 \mathrm{mV}$ (Zhao et al., 2005; Toyoda et al., 2009). Long-term depression (LTD) was induced using low frequency stimulation: 900 stimulation pulses at $1 \mathrm{~Hz}$. The occurrence of LTP or LTD was assessed by comparing the average EPSC amplitude from 5-10 $\mathrm{min}$ of the baseline to 25-30 min after the plasticity induction protocol (Nicoll, 2017). EPSC responses to electrically evoked paired pulse stimulation (50 ms intervals) were recorded after the $10 \mathrm{~min}$ baseline and again $30 \mathrm{~min}$ after the plasticity induction protocol. The paired pulse ratio (PPR) was obtained by averaging the mean amplitude of the second EPSC by that of the first (Kim and Alger, 2001). We recorded and analyzed 3 min long segments of mEPSCs in separate slices that did not undergo electrical stimulation. Autodetection parameters for inclusion of events was determined by calculating minimum threshold: root mean square $(\mathrm{RMS})^{2} \times 1.5$. To control for oversampling and unequal sample size between groups, we performed quantile sampling of mEPSC frequency and amplitude by computing 29 evenly spaced quantile values from each neuron, starting at the $1^{\text {st }}$ percentile and ending at the $94.2^{\text {th }}$ percentile, with a step size of $3.33 \%$ (Hanes et al., 2020). Data acquisition was performed using Clampex and analyzed with Clampfit (Molecular Devices). Mini Analysis software (Synaptosoft, USA) was used to analyze mEPSC recordings.

\section{Results}

\section{Perinatal fentanyl exposure}

We administered fentanyl citrate $(10 \mu \mathrm{g} / \mathrm{mL})$ in the drinking water of pregnant mouse dams throughout their pregnancy and until their litters were weaned at postnatal day (PD) 21 (Fig. 1A). We chose $10 \mu \mathrm{g} / \mathrm{ml}$ since it is the optimal concentration mice will readily self-administer without producing motor deficits (Wade et al., 2008, 2013) and is well below the mouse oral LD50 (fentanyl citrate MSDS, Cayman Chemical, Ann Arbor, MI). We have recently found that perinatal treatment with $10 \mu \mathrm{g} / \mathrm{ml}$ results in behavioral and synaptic deficits in adolescent mice (Alipio et al., 2021b). Offspring were weaned into either standard or enriched housing environments (Fig. 1, Table 1). 


\section{A}

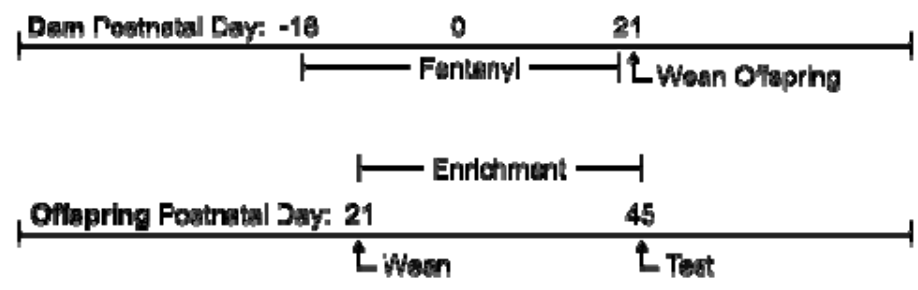

旦
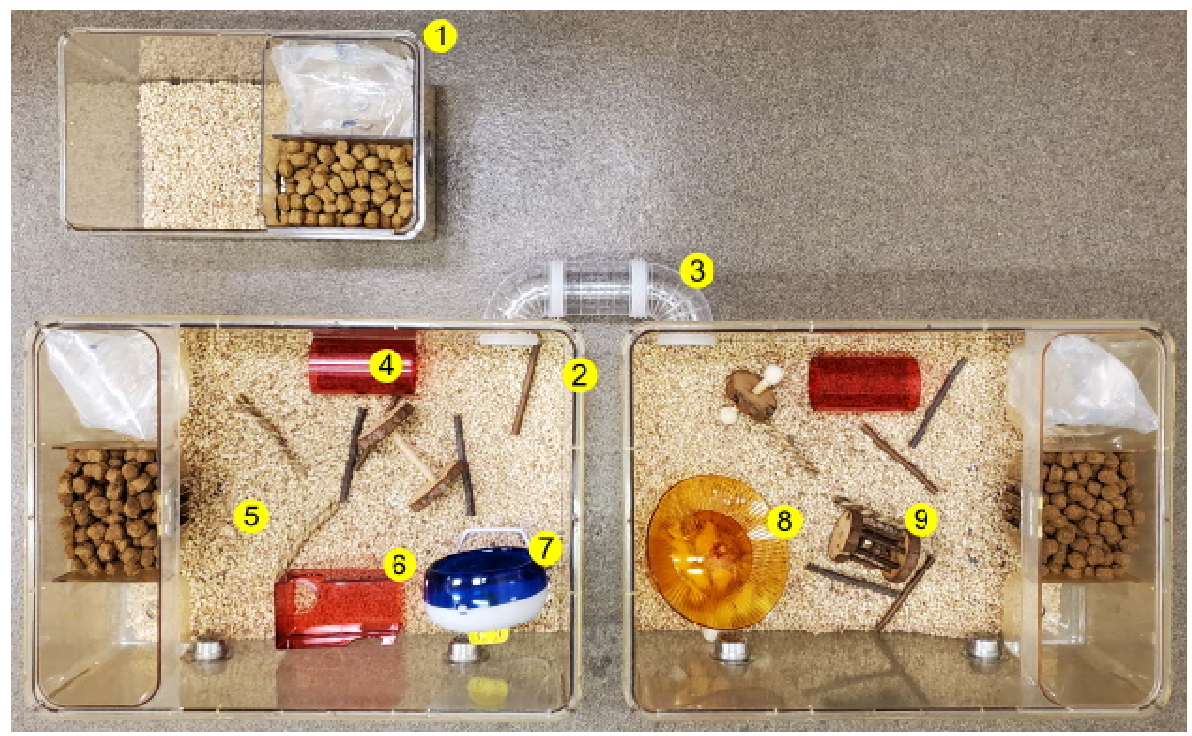

Figure 1. Experimental timeline of exposure to perinatal fentanyl and postnatal housing conditions. (A) Timeline depicting fentanyl exposure of mouse dams throughout pregnancy until weaning of offspring on postnatal day (PD) 21. (B) Offspring were weaned into standard (top cage) or enriched (bottom cage) housing conditions until behavioral (PD 45/47) and electrophysiological (PD 48-55) analyses took place. Enrichment items are numbered (1-9) and listed in Table 1.

Table 1 Product information for individual housing items

$\begin{array}{ll}\text { Item } & \text { Description } \\ 1 & \text { Standard housing } \\ 2 & \text { Environmental enrichment housing } \\ 3 & \text { Connecting tunnel } \\ 4 & \text { Tunnel } \\ 5 & 1 \text { " deep bedding } \\ 6 & \text { Platform } \\ 7 & \text { Standing running wheel } \\ 8 & \text { Dome and plate running wheel } \\ 9 & \text { Variety chew toys }\end{array}$

Product Info
Mouse $750^{\mathrm{TM}}$ ventilated cage
One Cage $2100^{\mathrm{TM}}$
DIY clear tunnel
Bio-Serv ${ }^{\mathrm{TM}}$ Mouse Tunnel
Corn Cob Bedding
Bio-Serv ${ }^{\mathrm{TM}}$ Mouse Retreat
Silent Spinner Wheel $4.5^{\prime \prime}$
Bio-Serv ${ }^{\mathrm{TM}}$ InnoDome and InnoWheel
Small animal chew toys

\section{Company}

Lab Products

Lab Products

POPETPOP

Fisher Scientific

Lab Supply

Fisher Scientific

Kaytee

Fisher Scientific

Niteangel

\section{Environmental enrichment reverses the sustained behavioral deficits that are induced by perinatal fentanyl exposure}


Hyperactivity. We used the open field test (Fig. 2A) to examine general locomotor behavior of adolescent male and female mice perinatally exposed to either vehicle or fentanyl, raised in either standard or an enriched housing environment (Fig. 2B-C; $n=15-20$ mice per group, about $50 / 50$ male to female ratio). There was no significant sex $\times$ drug $\times$ housing interaction $(F(1,59)$ $=2.83, p=0.09$ ) nor any differences between sex within each drug and housing group (Tukey's post hoc, $p>0.05$ ) on total distance traveled. Therefore, data from both sexes were grouped according to drug and housing condition. All mice habituated to the open field over time, in that distance traveled progressively decreased over the course of the procedure (Fig. 2B; $F$ ( 3.7 , $233.7)=119.70, p<10^{-4}, \mathrm{P \eta}^{2}=0.65$, large effect $)$. There was a significant time $\times$ drug (Threeway RM ANOVA, $F(5,310)=6.69, p<10^{-4}, \mathrm{P \eta}^{2}=0.09$, medium effect $)$ and drug $\times$ housing interaction $\left(F(1,62)=7.07, p=0.009, \mathrm{P}^{2}=0.10\right.$, medium effect $)$. There was a significant drug $\times$ housing interaction in total distance traveled (Fig. $2 \mathrm{C}$; Two-way ANOVA, $F(1,62)=7.07, p$ $=0.01, \mathrm{P}^{2}=0.10$, medium effect), with fentanyl-exposed, standard-housed mice exhibiting higher total distance traveled than vehicle-exposed, standard-housed mice (Tukey's post hoc, $p=$ 0.01 , Cohen's $d=1.23$, large effect). While environmental enrichment did not influence locomotor behavior on its own (i.e., in vehicle-exposed mice; Tukey's post hoc, $p=0.97$ ), it attenuated the hyperactivity of mice perinatally exposed to fentanyl (Tukey's post hoc, $p=$ 0.007 , Cohen's $d=1.58$, large effect). These data suggest that perinatal fentanyl exposure leads to hyperactivity and that environmental enrichment can reverse this effect without itself perturbing locomotor behavior.

Anxiety-like behavior. To test our prediction that environmental enrichment will attenuate anxiety-like behavior, we assessed time spent in the center zone during the open field test (Fig. 2D; $n=15-20$ mice per group; Kruskal-Wallis test, $H=10.17, p=0.01, \mathrm{P}^{2}=0.01$, small effect). Fentanyl-exposed, standard-housed mice spent less time in the center zone than did vehicleexposed, standard-housed mice (Dunn's post hoc, $p=0.02$, Glass' delta $=0.79$, medium effect), and this, too, was selectively reversed by environmental enrichment (Dunn's post hoc, $p=0.004$, Glass' delta $=0.88$, large effect). The observation that enrichment improves center-zone exploration of fentanyl-exposed mice is notable, given the sustained hyperlocomotor/anxiogenic effect induced by fentanyl under standard-housing conditions. This is further evidenced by fentanyl-exposed, standard-housed mice spending less time in the center zone over the course of the procedure relative to their enriched-housed fentanyl-exposed counterparts (Fig. 2E; KruskalWallis test, $H=14.80, p=0.002, \mathrm{P}^{2}=0.01$, small effect; Dunn's post hoc, $p=0.01$, Glass' delta $=1.15$, large effect), with the hyperlocomotor phenotype promoting center zone entries among fentanyl-exposed, standard-housed. These data suggest that environmental enrichment reduces anxiety-like behavior in adolescent mice that were perinatally exposed to fentanyl.

Sensory adaptation. We tested sensory adaptation, a reduction in sensitivity to repeated stimuli, by applying von Frey filaments to the plantar surface of the hind paw (Fig. 2F-H; $n=11-19$ mice per group). To test whether mice can sense tactile stimuli, we assessed hind paw withdrawal to threshold stimulation (Fig. 2G). There was no interaction nor main effect of drug and housing condition on paw withdraw threshold, suggesting that all mice similarly perceive tactile stimuli (Kruskal-Wallis test, $H=6.80, p=0.07$ ). Next, we tested whether mice adapt to repeated application of von Frey filaments above their threshold hind paw withdrawal response (Fig. 2H). There was a significant drug $\times$ housing interaction (Two-way ANOVA, $F(1,54)=56.27, p<$ $10^{-4}, \mathrm{P \eta}^{2}=0.51$, large effect). Fentanyl-exposed, standard-housed mice continued to respond to the stimuli more than twice as much as vehicle-exposed, standard-housed mice (Tukey's post 
hoc, $p<10^{-4}$, Glass' delta $=4.31$, large effect $)$. Enriched housing completely reversed this sensory maladaptation (Tukey's post hoc, $p<10^{-4}$, Glass' delta $=6.22$, large effect). These data suggest that perinatal fentanyl exposure leads to a sustained impairment in tactile sensory adaptation that can be reversed by environmental enrichment. Notably, while perinatal fentanyl exposure promotes hyperactivity and anxiety-like behavior relative to vehicle-exposed mice with large and medium effect, respectively, its influence on sensory adaptation was larger. These data suggest that fentanyl leads to an enduring sequalae of behavioral changes that can be fully reversed by environmental enrichment, with sensory maladaptation possibly being among the most prominent changes induced by perinatal opioid exposure.

A
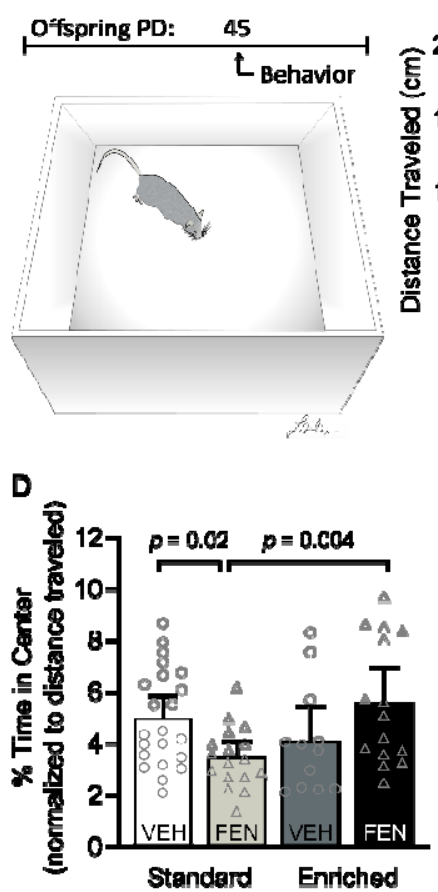

F

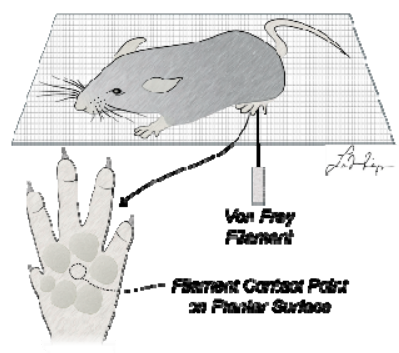

B

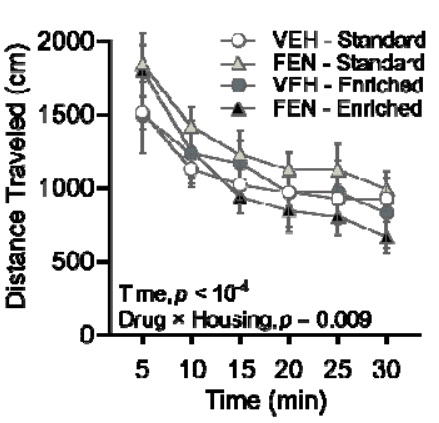

E

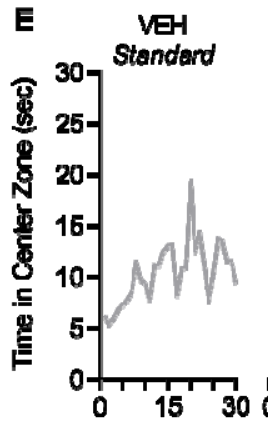

c

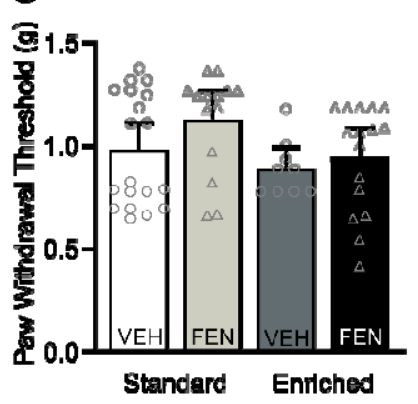

C

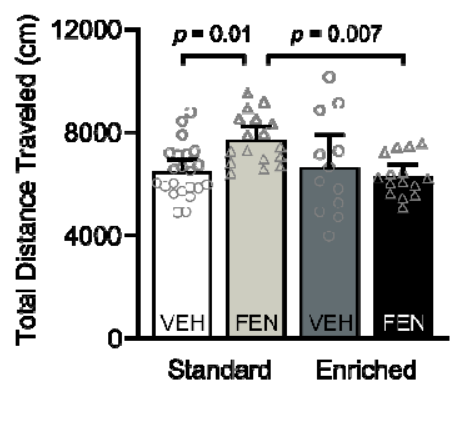

FEN

Standerd

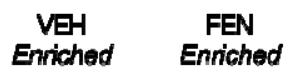

Enriched

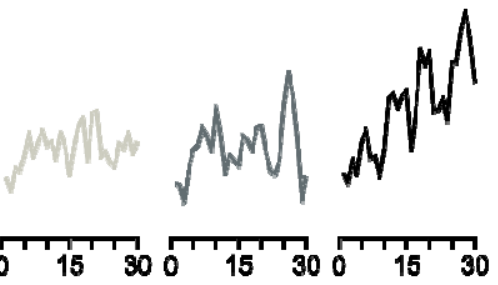

H

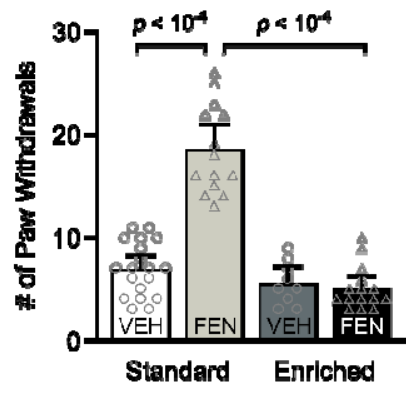


Figure 2. Environmental enrichment reverses the sustained behavioral deficits that are induced by perinatal fentanyl exposure. (A) Timeline depicting behavioral assays and a schematic of the open field test. $n=15-20$ mice per group. (B, C) Perinatal fentanyl exposure increases distance traveled across time. (D, E) Perinatal fentanyl exposed mice raised with environmental enrichment have comparable distance traveled with vehicle controls but spend less time in the center area of the open field. Fentanyl-exposed mice raised with environmental enrichment spend more time in the center area of the open field, comparable to vehicle controls. (F) Graphic depicting von Frey tactile stimulation on the plantar surface of the hind paw. (G) There were no differences across groups in the paw withdrawal threshold to tactile stimulation. $n=11-19$ mice per group. (H) Standard housed fentanyl exposed mice have a higher number of paw withdrawal responses to repeated stimuli than standard housed vehicle mice. Fentanyl exposed mice raised with environmental enrichment have a lower number of paw withdrawal responses, comparable to vehicle mice raised in standard and enriched environment. Data depict means, $p$ values, and 95\% confidence intervals.

\section{Environmental enrichment restores long-term potentiation in S1 layer 2/3 neurons after perinatal fentanyl exposure}

The fentanyl-induced impairment in sensory adaptation, as well as its reversal by environmental enrichment, suggests that these changes may be associated with altered synaptic plasticity in somatosensory cortex (S1). To test this, we assessed whether perinatal fentanyl exposure and environmental enrichment influenced synaptic potentiation of excitatory postsynaptic currents (EPSCs) in S1 layer 2/3 neurons (Fig. 3; $n=6-8$ mice/group, 1 neuron/mouse, about 50/50 male to female ratio). There was no significant sex $\times \operatorname{drug} \times$ housing interaction $(F(1,21)=1.21, p=$ 0.28 ) nor any differences between sex within each drug and housing group (Tukey's post hoc, $p$ $>0.05$ ) on long-term plasticity. Therefore, data from both sexes were grouped according to drug and housing condition.

Mechanisms that govern the expression of short-term potentiation (STP) and long-term potentiation (LTP) differ (Schulz and Fitzgibbons, 1997), and both may be involved in sensory adaptation behavior (Monday et al., 2018; Motanis et al., 2018), therefore we assessed both forms of synaptic potentiation among our groups. Both STP and LTP were readily induced in S1 layer 2/3 neurons from vehicle-exposed, standard-housed mice (Fig. 3A). Amplitudes of evoked EPSCs were higher at both STP 0-5 min (Bonferroni's post hoc, $p=0.008$, Cohen's $d=1.86$, large effect) and LTP 25-30 min (Bonferroni's post hoc, $p=0.006$, Cohen's $d=2.63$, large effect) timepoints after the LTP paired induction protocol (80 electrical stimulation pulses at 2 $\mathrm{Hz}$ paired with postsynaptic depolarization to $+30 \mathrm{mV}$ ) relative to baseline $-5-0 \mathrm{~min}$ (One-way RM ANOVA, $F(2,12)=8.71, p=0.004, \mathrm{P}^{2}=0.59$, large effect $)$. In contrast, neurons from fentanyl-exposed, standard-housed mice did not potentiate, and instead showed a long-term depression (LTD)-like effect with significantly reduced EPSC amplitudes at the 25-30 min timepoint (Bonferroni's post hoc, $p=0.01$, Cohen's $d=1.92$, large effect) relative to baseline (Fig. 3B; One-way RM ANOVA, $F(2,12)=5.47, p=0.02, \mathrm{P}^{2}=0.48$, large effect). In vehicleexposed mice raised with environmental enrichment, the induction parameters tested here failed to evoke a statistically significant change in EPSC amplitude from baseline (Fig. 3C; One-way RM ANOVA, $F(2,10)=4.38, p=0.04, \mathrm{P}^{2}=0.46$, large effect; Bonferroni's post hoc, $p>$ 0.05). In fentanyl-exposed mice raised with environmental enrichment, the LTP-induction 
protocol produced STP at the 0-5 min timepoint (Bonferroni's post hoc, $p=0.04$, Cohen's $d=$ 1.42, large effect), but EPSC amplitude was not significantly different from baseline by 25-30 min post-induction (Fig. 3D; One-way RM ANOVA, $F(2,14)=3.27, p=0.06$ ).

Consistent with these within-group comparisons, between-group comparisons revealed a complete block of STP and LTP in neurons from fentanyl-exposed, standard-housed mice and a restoration of STP and LTP in fentanyl-exposed mice by environmental enrichment, compared to their respective controls (Fig. 3E). There was a significant time $\times$ drug $\times$ housing interaction (Three-way RM ANOVA, $F(2,50)=11.43, p<10^{-4}, \mathrm{P}^{2}=0.31$, large effect). At the $0-5$ min timepoint, neurons from vehicle-exposed, enriched-housed mice had lower post-LTP EPSC amplitudes (Tukey's post hoc, $p<10^{-3}$, Cohen's $d=1.75$, large effect) than their vehicleexposed, standard-housed counterparts. Neurons from fentanyl-exposed, standard-housed mice had lower EPSC amplitudes than vehicle-exposed, standard-housed mice (Tukey's post hoc, $p<$ $10^{-4}$, Cohen's $d=2.28$, large effect). This reduction in fentanyl-exposed mice was restored by environmental enrichment (Tukey's post hoc, $p=0.01$, Cohen's $d=2.02$, large effect). At the LTP 25-30 min timepoint, neurons from fentanyl-exposed, standard-housed mice had lower EPSC amplitudes than vehicle-exposed, standard-housed mice (Tukey's post hoc, $p<10^{-4}$, Cohen's $d=3.22$, large effect). This reduction was also restored by environmental enrichment (Tukey's post hoc, $p=0.04$, Cohen's $d=1.33$, large effect).

While nearly all neurons from vehicle-exposed, standard-housed mice exhibited STP (Fig. 3F; 5/7 neurons, 71.4\%) and LTP (6/7 neurons, 85.7\%), none of the neurons from fentanyl-exposed, standard-housed mice exhibited STP or LTP (0/7 neurons, 0\%). Neurons from vehicle-exposed, enriched-housed mice did not exhibit STP (0/6 neurons, $0 \%)$, however, half of the neurons exhibited LTP (3/6 neurons, 50\%). Neurons from fentanyl-exposed enriched-housed mice exhibited both STP and LTP (5/8 neurons, 62.5\%). Between-group comparisons further indicate that fentanyl-exposed, standard-housed mice had a lower occurrence of both STP and LTP than vehicle-exposed, standard-housed mice (Fisher's exact test, STP, $p=0.03$, odds ratio $=33$, large effect ; LTP, $p=0.01$, odds ratio $=65$, large effect ) and that environmental enrichment increased the proportion of neurons that exhibit STP and LTP in fentanyl-exposed mice compared to fentanyl-exposed, standard-housed mice (Fisher's exact test, STP, $p=0.02$, odds ratio $=23.57$, large effect; LTP $p=0.02$, odds ratio $=23.57$, large effect). These data demonstrate that perinatal fentanyl exposure impairs both STP and LTP in S1 layer 2/3 neurons from mice raised under standard housing conditions, and that environmental enrichment can restore potentiation to levels comparable to vehicle-exposed, standard-housed mice. Neurons from control mice raised in an enriched environment exhibit an attenuated and lower occurrence of STP and LTP relative to standard housing conditions, which may suggest an occlusive effect of enrichment on synaptic plasticity. 

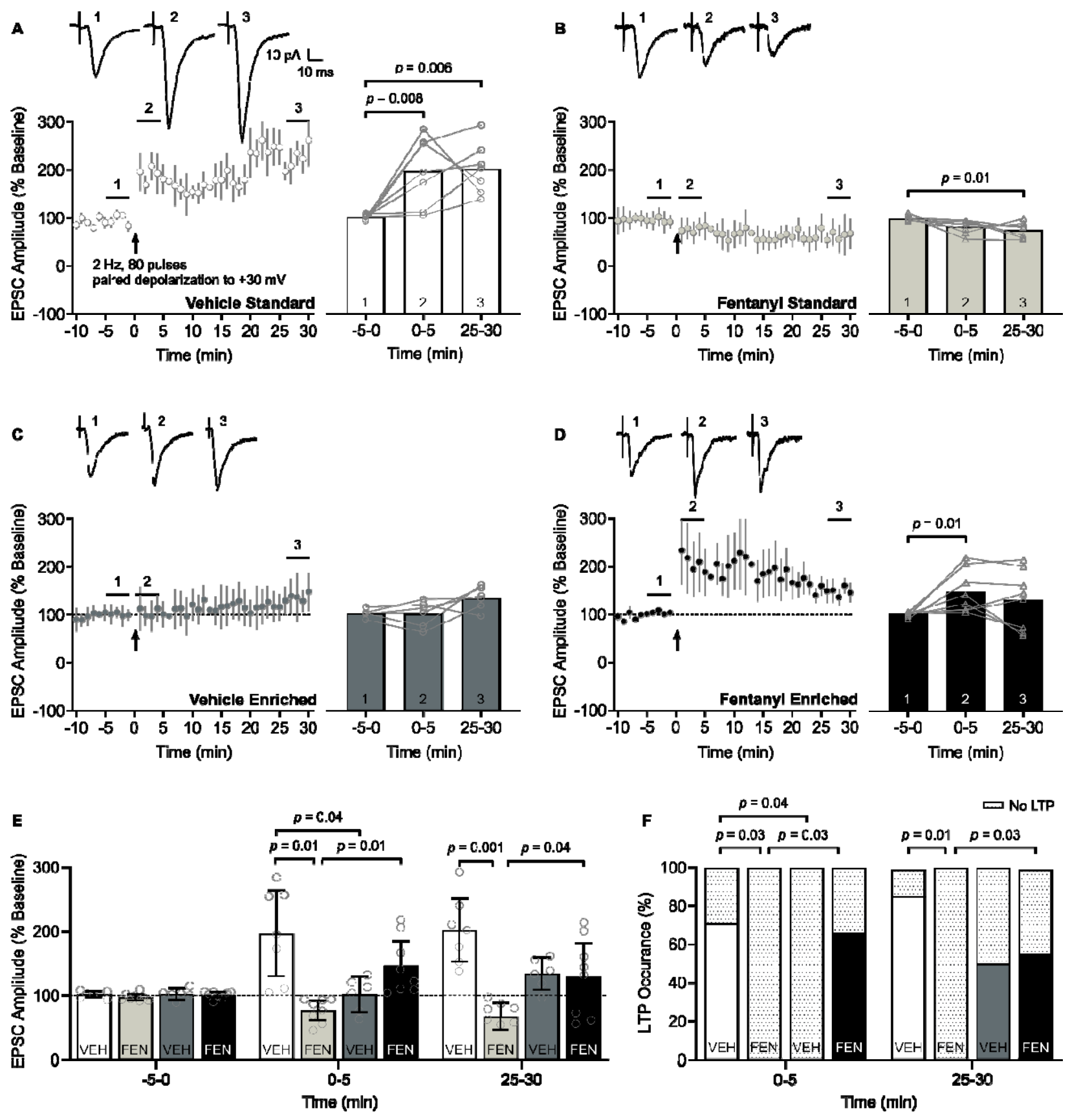
Figure 3. Environmental enrichment restores potentiation in S1 layer 2/3 neurons after perinatal fentanyl exposure (A-D) Time course (left) of short- and long-term potentiation (STP/LTP) of excitatory postsynaptic currents (EPSCs) following paired induction (80 electrical stimulation pulses at $2 \mathrm{~Hz}$ paired with postsynaptic depolarization to $+30 \mathrm{mV})$. Bar graphs (right) show group data comparing EPSC amplitudes at baseline -5-0 min (1), STP 05 min (2), and LTP 25-30 min (3) after LTP induction. Insets depict sample traces from times indicated on time course graph. (A) Within-group comparisons indicate STP and LTP were induced in S1 layer 2/3 neurons from vehicle-exposed, standard-housed mice. (B) LTP paired induction parameters induced long-term depression (LTD) of the EPSC in fentanylexposed, standard-housed mice. (C) LTP paired induction protocol failed to further potentiate EPSC amplitude from vehicle-exposed, enriched-housed mice. (D) The paired induction protocol induced STP, but not LTP, in fentanyl-exposed, enriched-housed mice. (E) Between-group comparisons of the EPSC amplitudes post LTP induction. Fentanylexposed, standard-housed mice had lower EPSC amplitudes than vehicle-exposed, standardhoused mice at 0-5 and 25-30 min timepoints. Environmental enrichment increased the EPSC amplitude of fentanyl-exposed, enriched-housed mice, relative to their fentanylexposed, standard-housed counterparts also at 0-5 and 25-30 min timepoints. (F) Betweengroup comparisons indicate fentanyl-exposed, standard-housed mice had a lower occurrence of neurons that exhibit STP and LTP than vehicle-exposed, standard-housed mice. Raising fentanyl-exposed mice in environmental enrichment increased the proportion of neurons that potentiate. $n=6-8$ mice/group, 1 neuron/mouse. Data depict means and 95\% confidence intervals.

\section{Environmental enrichment suppresses long-term depression}

We assessed whether perinatal fentanyl exposure influenced LTD of EPSCs in S1 layer 2/3 neurons and the impact of environmental enrichment on these changes (Fig. 4; $n=5-6$ mice/group, 1 neuron/mouse). LTD of EPSC amplitudes were evident following low-frequency electrical stimulation (900 stimuli, $1 \mathrm{~Hz}$ ) in S1 layer 2/3 neurons from vehicle-exposed, standardhoused mice (Fig. 4A; Paired $t$-test, $p<10^{-4}$, Cohen's $d=11.13$, large effect) and in fentanylexposed, standard-housed mice (Fig. 4B; Wilcoxon matched-pairs signed rank test, $p=0.03$, Glass' delta $=40.11$, large effect $)$. However, LTD was not induced in neurons from vehicleexposed mice raised in an enriched environment (Fig. 4C; Wilcoxon matched-pairs signed rank test, $p=0.12$ ). In contrast, LTD was readily induced in fentanyl-exposed, enriched-housed mice (Fig. 4D; Wilcoxon matched-pairs signed rank test, $p=0.03$, Glass' delta $=6.46$, large effect).

Consistent with this, between-group comparisons of post-LTD EPSC amplitudes revealed a significant drug $\times$ housing interaction (Fig. 4E; Two-way ANOVA, $F(1,18)=5.86, p=0.02$, $\mathrm{P \eta}^{2}=0.24$, large effect) with greater LTD in neurons from vehicle-exposed, standard-housed mice, relative to vehicle-exposed, enriched-housed mice (Tukey's post hoc, $p=0.006$, Cohen's $d$ $=5.26$, large effect). All neurons from vehicle-exposed standard-housed mice exhibited LTD (5/5 neurons) whereas only $20 \%$ of neurons from vehicle-exposed, enriched-housed mice exhibited LTD (Fig. 4F; 1/5 neurons; Fisher's exact test, $p=0.04$, odds ratio $=40$, large effect). Most neurons from fentanyl-exposed mice exhibited LTD (5/6 neurons 83.33\%) in either housing condition, respectively. 
These data demonstrate that perinatal fentanyl exposure does not appear to affect LTD induction or expression, regardless of housing conditions. Similar to LTP, LTD is readily induced in S1 layer $2 / 3$ neurons of mice raised in standard, conventional cages, but not in neurons from mice raised in an enriched environment.
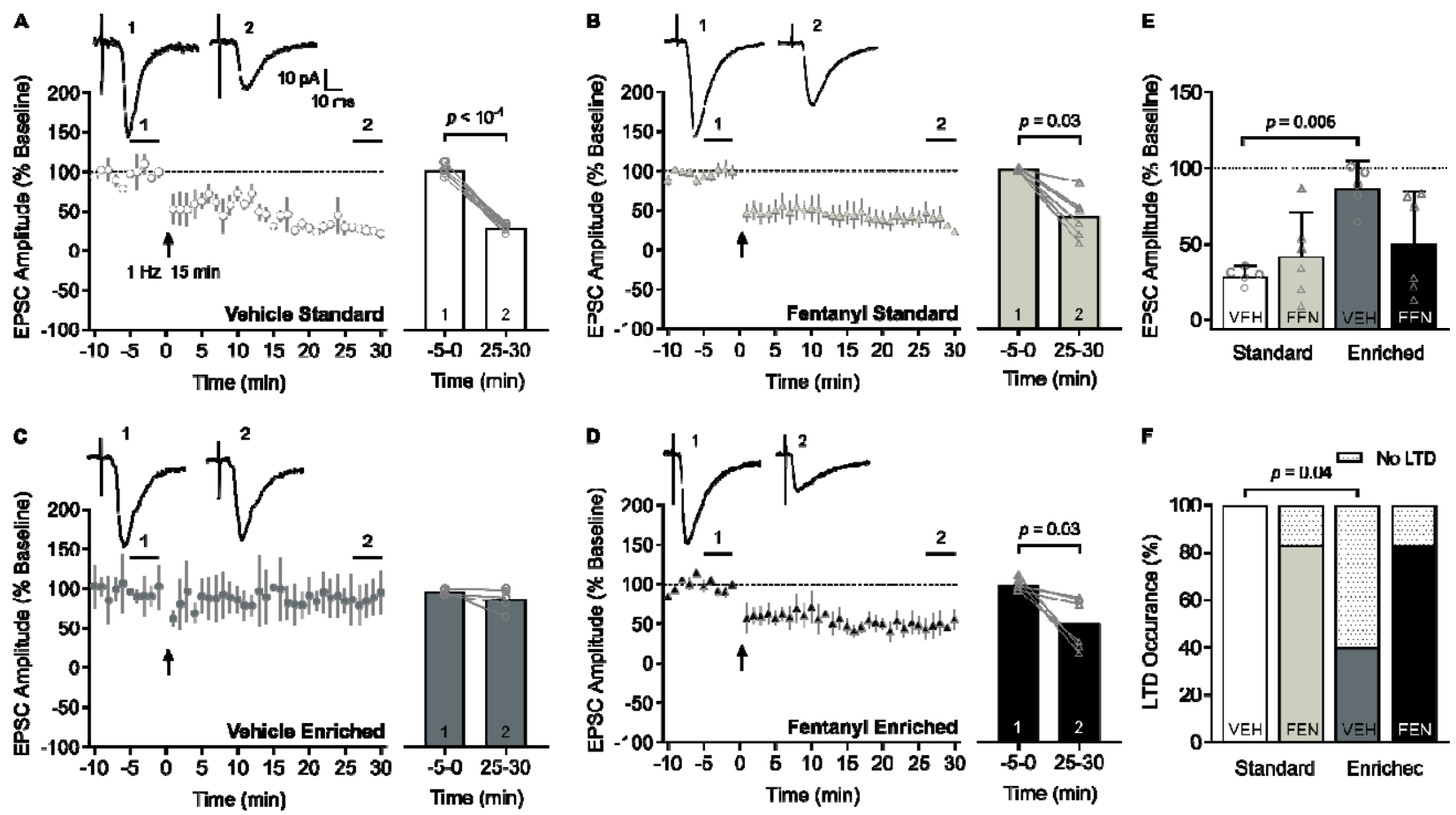

Figure 4. Environmental enrichment suppresses long-term depression. (A-D) Time course (left) of long-term depression (LTD) of excitatory postsynaptic currents (EPSCs) following low frequency stimulation (900 electrical stimulation pulses at $1 \mathrm{~Hz}$ ). Bar graphs (right) show group data comparing EPSC amplitudes at baseline (1) and at 25-30 min after LTD induction (2). Insets depict sample traces from times indicated on time course graph. (A) Within-group comparisons indicate LTD was induced in S1 layer 2/3 neurons from vehicle-exposed, standard-housed mice and in (B) fentanyl-exposed, standard-housed mice. (C) LTD was not induced in vehicle-exposed, enriched-housed mice, but was in (D) fentanyl-exposed, enrichedhoused mice. (E) Between-group comparisons of the EPSC amplitudes post-LTD induction indicate that vehicle-exposed, enriched-housed mice failed to induce LTD compared to their vehicle-exposed, standard-housed counterparts. (F) Between-group comparisons of the occurrence of LTD indicate vehicle-exposed, enriched-housed mice had a lower occurrence of neurons that exhibit LTD than vehicle-exposed, standard-housed mice. $n=5-6$ mice/group, 1 neuron/mouse. Data depict means and $95 \%$ confidence intervals.

\section{Evoked glutamate release probability is not influenced by perinatal fentanyl exposure or environmental enrichment}

We assessed paired pulse ratios (PPRs) to determine if the changes in plasticity induced by perinatal fentanyl exposure and environmental enrichment were mediated by presynaptic changes in vesicle release probability (Fig. 5). Figure 5A depicts sample traces from each of the experimental groups. Before LTP/LTD induction, there were no differences in baseline PPR among the experimental groups (Fig. 5B; $n=9-12$ mice/group, 1 neuron/mouse; Kruskal-Wallis 
test, $p>0.05$ ), nor did PPR change following the induction of LTP (Fig. 5C: $n=4-6$ mice/group, 1 neuron/mouse; Paired $t$-test, $p>0.05$ ) or LTD (Fig. 5D: $n=4-6$ mice/group, 1 neuron/mouse; Paired $t$-test, $p>0.05)$. These data suggest that neither perinatal fentanyl exposure nor environmental enrichment influence the probability of evoked glutamate release at baseline or following induction of LTP or LTD in S1 layer 2/3 neurons with the induction protocols used in the current study.
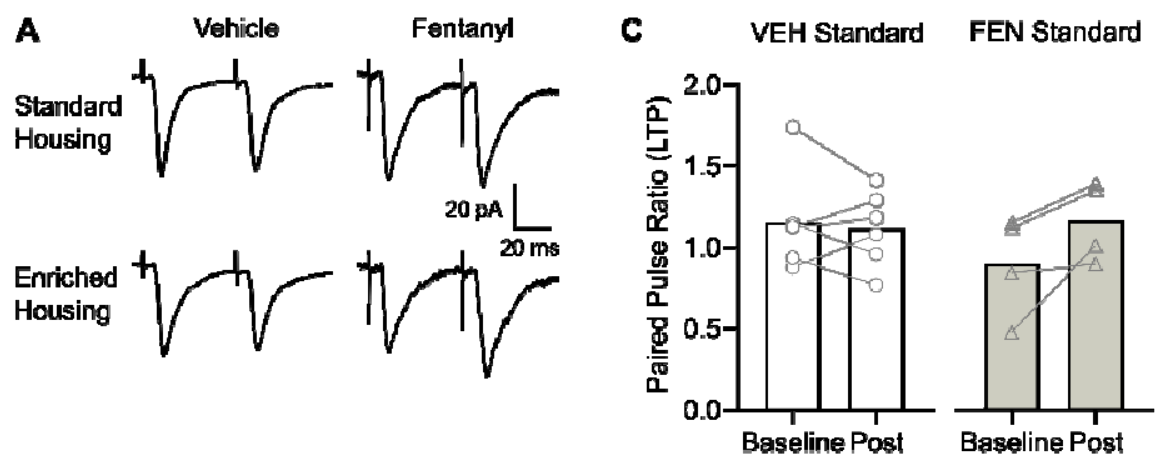

VEH Enriched

FEN Enriched
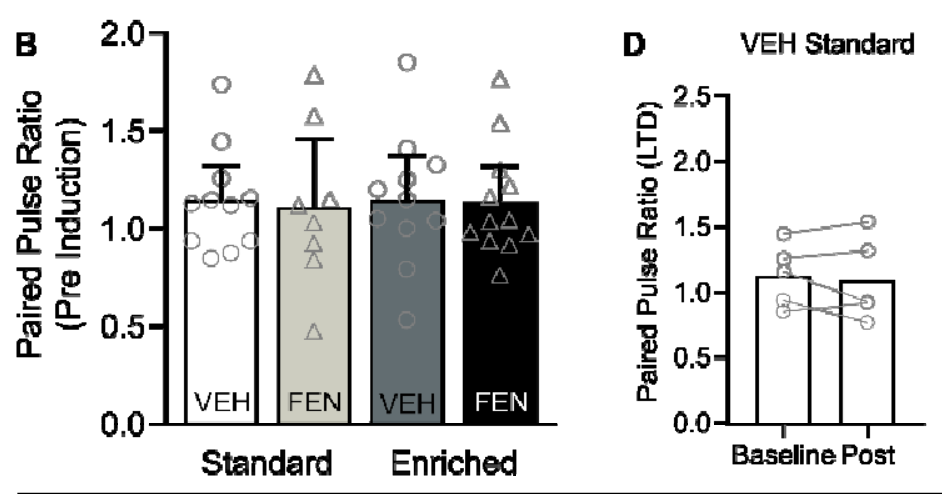

FEN Standard
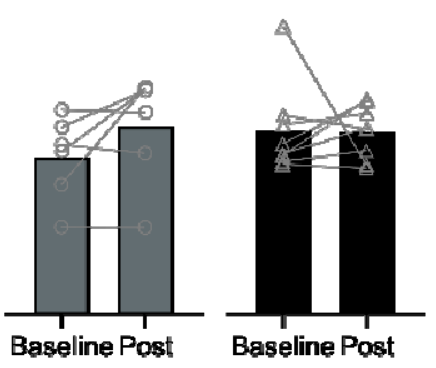

Figure 5. Evoked glutamate release probability is not influence by perinatal fentanyl exposure or environmental enrichment. (A) Representative traces of EPSC responses to paired pulse stimulation. (B) There were no differences in the paired pulse ratio (PPR) between groups prior to plasticity induction ( $n=9-12 \mathrm{mice} /$ group, 1 neuron/mouse). (C, D) Within each group, there were no differences in baseline PPR compared to PPR post-LTP or LTD induction ( $n=4-6$ mice/group, 1 neuron/mouse). Data depict means and 95\% confidence intervals.

\section{Environmental enrichment restores changes in mEPSC frequency induced by perinatal fentanyl exposure}

Some of the mechanisms and functions of spontaneous synaptic transmission are distinct from that of action potential-evoked synaptic transmission (Kavalali, 2015). To determine if perinatal fentanyl exposure influenced spontaneous excitatory synaptic transmission, we recorded miniature excitatory postsynaptic currents (mEPSCs) in S1 layer 2/3 neurons (Fig. 6; $n=6-8$ mice/group, 1-2 neurons/mouse). We averaged all events from each neuron and animal for between-group comparisons of mEPSC frequency (Fig 6B; Kruskal-Wallis test, $H=12.41, p=$ $0.006, \mathrm{P}^{2}=0.03$, medium effect ). Neurons from vehicle-exposed, enriched-housed mice had 
higher mEPSC frequency than vehicle-exposed, standard-housed mice (Dunn's post hoc, $p=$ 0.01 , Glass' delta $=21.00$, large effect ).

Given that averaging mEPSC events can be confounded by cell-to-cell differences in vesicle release dynamics and/or postsynaptic cluster sizes (Wu et al., 2007), we further assessed these differences in mEPSC frequency by examining their cumulative probability (Fig. 6C; KruskalWallis test, $H=42.61, p<10^{-4}, \mathrm{P \eta}^{2}<0.008$, small effect ). Since we observed measurable differences among the groups in the total number and range of release events, we used a quantile-based approach described by Hanes et al., 2020 (Hanes et al., 2020) to control for these unequal distributions. Neurons from fentanyl-exposed, standard-housed mice had higher mEPSC frequency than neurons from vehicle-exposed, standard-housed mice (Dunn's post hoc, $p<10^{-4}$, Glass' delta $=2.98$, large effect) and environmental enrichment normalized this increase in mEPSC frequency (Dunn's post hoc, $p=0.01$, Glass' delta $=0.52$, medium-effect). Environmental enrichment also enhanced mEPSC frequency in vehicle-treated conditions relative to neurons from mice raised in standard housing (Dunn's post hoc, $p<10^{-4}$, Glass' delta $=3.34$, large effect). In contrast, there was no significant interaction nor main effect on the average (Fig. 6D; Two-way ANOVA, $p>0.05$ ) or cumulative probability (Fig. 6E; KruskalWallis test, $p>0.05$ ) of mEPSC amplitudes among the groups. These data suggest that both environmental enrichment and perinatal fentanyl exposure, independently establishes a higher frequency of miniature excitatory release at this synapse. Among perinatal fentanyl exposed mice, environmental enrichment restores the mEPSC frequency to a more quiescent state, resembling that of vehicle-exposed, standard-housed mice. 
A
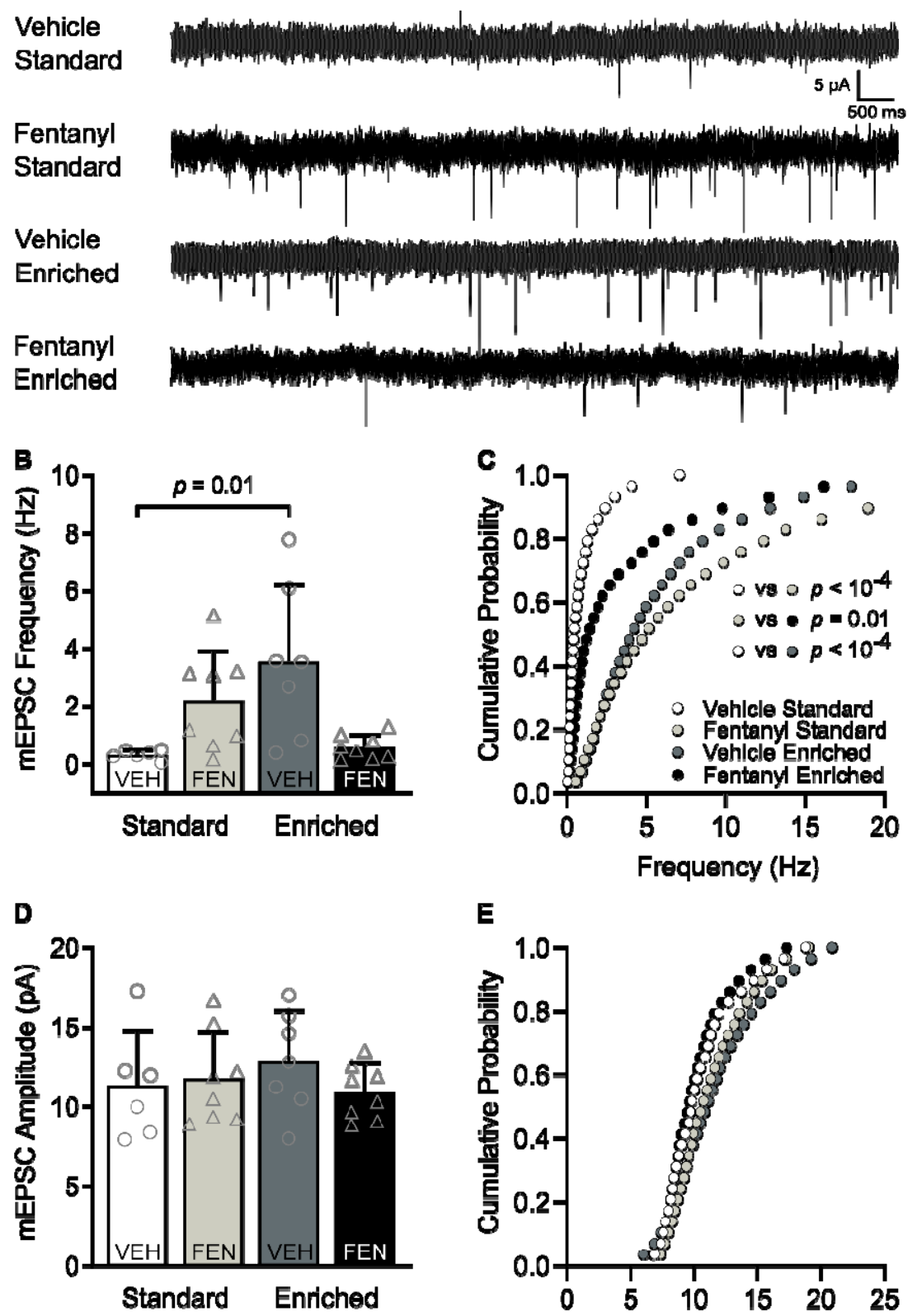

E

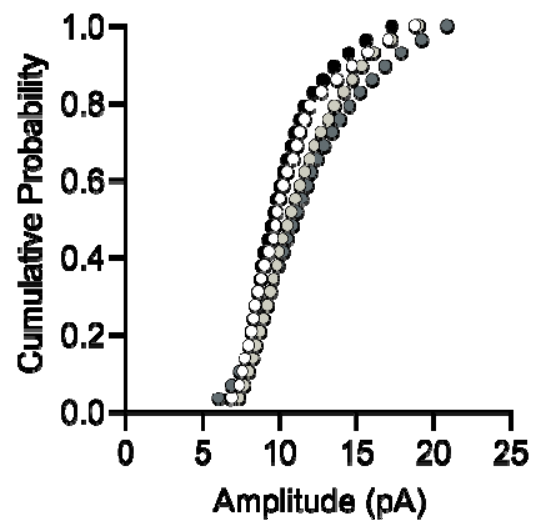

Figure 6. Environmental enrichment restores changes in mEPSC frequency induced by perinatal fentanyl exposure. (A) Representative traces of miniature excitatory postsynaptic currents (mEPSCs). (B) Grouped data of the averaged events from each neuron and animal indicating that mEPSC frequency is higher after environmental enrichment, compared to controls. (C) Cumulative probability curves of the frequency suggest perinatal fentanyl exposure results in increased mEPSC frequency, and that raising fentanyl-exposed mice with environmental enrichment reverses this increase. Enrichment also increased mEPSC frequency of vehicle control mice independent of perinatal fentanyl exposure. (D, E) There were no differences in mEPSC amplitudes. $n=6-8$ mice/group, 1-2 neurons/mouse. Data depict means, and $95 \%$ confidence intervals. 


\section{Discussion}

We hypothesized that environmental enrichment would mitigate the behavioral and synaptic deficits of perinatal fentanyl exposure. We administered fentanyl citrate in the drinking water of pregnant mouse dams throughout their pregnancy and until their litters were weaned at postnatal day (PD) 21. We acknowledge that opioid use among women likely occurs prior to pregnancy and contributes to both short- and long-term deleterious outcomes (Byrnes and Vassoler, 2018). To avoid effects on sexual behavior and conception (Johnson et al., 2011), we began fentanyl exposure after copulatory plugs were identified. This exposure paradigm increases litter mortality rate and decreases litter size without influencing maternal care behavior, recapitulating opioid use among pregnant women (Alipio et al., 2021b). Some studies stop opioid exposure at or a few days post parturition (Byrnes and Vassoler, 2018). We chose to continue fentanyl exposure until weaning because human cortical development at birth is equivalent to several days beyond PD 11 in mice (Clancy et al., 2001). Additionally, we wanted to avoid confounding effects due to changes in maternal care behavior, which can occur if opioid administration to the dam is discontinued following birth (Schlagal et al., 2021).

Consistent with our prediction, environmental enrichment attenuated the behavioral aberrations induced by perinatal fentanyl exposure, including hyperactivity, anxiety-like behavior, and sensory maladaptation. Enrichment also normalized short- and long-term potentiation (STP/LTP) and spontaneous excitatory synaptic transmission in primary somatosensory (S1) layer 2/3 neurons from perinatal fentanyl exposed mice. Notably, in naïve control mice, environmental enrichment results in attenuated STP/LTP and long-term depression (LTD), as well as increased spontaneous excitatory synaptic transmission.

\section{Behavior}

We previously showed that perinatal fentanyl exposure results in spontaneous somatic withdrawal signs, lasting anxiety-like behavior, and sensory maladaptation without influencing the dam's weight, food and liquid intake - recapitulating neonatal opioid withdrawal syndrome (Alipio et al., 2021b, 2021 a). Here, we expand upon our model by showing that perinatal fentanyl exposure leads to hyperactivity, a prominent feature of attention-deficit hyperactivity disorder (Montarolo et al., 2019), which is often diagnosed in children that were exposed to opioids perinatally (Schwartz et al., 2021).

\section{Synaptic plasticity}

Because perinatal fentanyl exposure led to impairments in sensory-related processing, we investigated changes in the efficacy of synaptic transmission in S1 layer 2/3. Plasticity in S1 layer $2 / 3$ is associated with sensory adaptation and rapid storage of immediate sensory memories (Brecht, 2017). Since there is an immediate temporal requirement for sensory adaptation, we predicted that plasticity in this region is associated with adaptive tactile behavior. Whereas LTP was readily induced by pairing repeated stimuli with postsynaptic depolarization of S1 layer 2/3 neurons, this LTP induction protocol failed to induce LTP in neurons from fentanyl-exposed mice. In contrast, perinatal fentanyl exposure did not influence the induction of LTD in response to low frequency stimulation. These results are consistent with previous studies showing that prenatal exposure to morphine suppresses LTP in the dentate gyrus of juvenile rats (Niu et al., 2009; Ahmadalipour et al., 2018). 
Here, we find that environmental enrichment increased the short-term potentiation of EPSCs from fentanyl-exposed mice. The potentiation was sustained in a subset of neurons up to $30 \mathrm{~min}$ post stimulation. We recorded out to 30 min post stimulation, which is within the accepted parameters of conventional LTP (Nicoll, 2017), to avoid possible confounding effects of a sustained timecourse (Abbas et al., 2015; Baltaci et al., 2019). We did not assess short-term depression since the resulting EPSC amplitude depression was sustained at least to 30 min post stimulation. Further studies are needed to investigate late phases of plasticity under these conditions.

\section{Synaptic activity}

We have previously reported that perinatal fentanyl exposure suppresses evoked and spontaneous glutamate release onto S1 layer 5 neurons, and enhances glutamate release onto anterior cingulate cortex (ACC) layer 5 neurons (Alipio et al., 2021b). Here we find that fentanyl exposure enhances spontaneous glutamate release onto $S 1$ layer $2 / 3$ neurons. That neurons in different cortical layers and areas express differences in the lasting effects of perinatal fentanyl exposure likely reflects differences in cortical developmental trajectory. Our data suggest that neurons that develop earlier (S1 layer 2/3 neurons) express a lasting increase in excitatory synaptic activity, whereas later developing neurons (S1 and ACC layer 5 neurons) exhibit decreased synaptic activity.

The different effects on spontaneous versus evoked glutamate release is not surprising. Mechanisms that regulate these two phenomena are well documented (Ramirez and Kavalali, 2011; Kavalali, 2015). For example, brain-derived neurotrophic factor (BDNF) enhances mEPSCs but not evoked EPSCs in immature cortical neurons (Taniguchi et al., 2000). We have previously found that perinatal fentanyl exposure decreases mRNA expression of TrkB, a receptor for BDNF (Alipio et al., 2021b). Given the differential expression patterns of TrkB across cortical layers (Cabelli et al., 1996), it is possible that our fentanyl exposure paradigm interferes with the developmental trajectory of TrkB expression and subsequently impairs cortical circuit function.

\section{Environmental enrichment effects on behavior}

Environmental enrichment restored the hyperactivity induced by perinatal fentanyl exposure, as well as the increase in anxiety-like behavior in a novel open field environment. This is consistent with studies demonstrating that rearing rodents in an enriched environment improves maladaptive phenotypic changes in preclinical models of ADHD and anxiety (Botanas et al., 2016; Korkhin et al., 2020; Yazdanfar et al., 2021). Environmental enrichment also restored the sensory maladaptation induced by perinatal fentanyl exposure. These findings might be relevant to addressing the sensory-related deficits in children that were perinatally exposed to opioids.

Other preclinical studies have shown that environmental enrichment can ameliorate behavioral changes induced by perinatal exposure to other drugs of abuse, including nicotine, cocaine, morphine, ethanol, antiadrenergic, and antihypertensive drugs (Ryan and Pappas, 1990; DowEdwards et al., 2014; Mychasiuk et al., 2014; Ahmadalipour et al., 2018; Wille-Bille et al., 2020; Yazdanfar et al., 2021). These results suggest that enrichment may benefit infants and children with such exposures. Importantly, our data also support that environmental enrichment may be a favorable intervention during early development, since it does not produce untoward behavioral outcomes on its own, at least not in the behavioral outcomes assessed in the current study. 


\section{Environmental enrichment effects on synaptic plasticity}

Environmental enrichment restored the perinatal fentanyl exposure-induced impairment in LTP in S1 layer 2/3 neurons. This is consistent with findings that environmental enrichment restores stress-induced reduction of LTP in hippocampal dentate gyrus and prefrontal cortical neurons, and that enrichment does not further increase LTP in non-stressed controls (Wang et al., 2020; Wu and Mitra, 2020).

We also found that environmental enrichment suppressed the ability of S1 layer 2/3 neurons to express either LTP or LTD. These findings are consistent with previous reports showing that environmental enrichment-induced plasticity occludes further potentiation of S1 layer 2/3 and 4 neurons (Mégevand et al., 2009). Similarly, enrichment reduces or blocks the induction of LTP in the dentate gyrus (Feng et al., 2001; Irvine et al., 2006; Eckert et al., 2010), perhaps by occluding LTP (Foster et al., 1996). The occlusion of experimentally driven plasticity by sensory experience is consistent with findings that cortical neurons may reach a modification ceiling following training on a sensorimotor task (Rioult-Pedotti et al., 2000). However, despite occlusion of LTP by natural behavior, further potentiation remains possible through other synaptic strengthening mechanisms (Clem et al., 2008). It remains to be determined if further plasticity can be induced under the conditions here, perhaps by way of different induction protocols.

\section{Environmental enrichment effects on synaptic activity}

Environmental enrichment restored the amplified spontaneous excitatory synaptic transmission in S1 layer 2/3 neurons induced by perinatal fentanyl exposure, and increased it in control mice. The contrasting effects of enrichment on fentanyl and on control mice reflect the multifactorial mechanisms in which enrichment influences cortical synaptic transmission (Baroncelli et al., 2010). Relevant findings demonstrate that environmental enrichment restores the decreased mEPSC frequency in S1 layer 2/3 neurons induced by sensory deprivation, and increases mEPSC frequency in control, non-deprived mice (Zheng et al., 2014). The mechanisms involved in the increased excitatory synaptic activity induced by perinatal fentanyl exposure and the restoration by environmental enrichment has yet to be determined.

Converging evidence from longitudinal human studies and preclinical models demonstrate that perinatal exposure to opioids results in long-lasting molecular, circuit, network, and behavioral aberrations. Current treatment options are aimed at relieving acute symptoms exhibited by newborns with neonatal opioid withdrawal. No such treatments are available for the developing child or adolescent. Our results provide insights into the underlying circuit changes involved in the lasting anxiety and sensory-related deficits induced by perinatal fentanyl exposure, and suggest that environmental enrichment may be leveraged to ameliorate or reverse the lasting deleterious effects of this early opioid exposure.

\section{Acknowledgements}

JBA and LMR conceptualized the project, designed and performed the experiments, analyzed and interpreted the data, and co-wrote the manuscript; MP assisted in data analysis; AK supervised the research, performed analyses, and co-wrote the manuscript. We thank Urja Kuppa for preliminary analysis of miniature excitatory postsynaptic currents. 


\section{Funding and Disclosures}

This research was supported by the Opioid Use Disorders Initiative: MPowering the State, from the State of Maryland (to AK), NIH/NIDA F31-DA051113 (to JBA), NIH/NIGMS R25-

GM055036 (to JBA and LMR), NIH/NIMH F31-MH123066 (to LMR), NIH/NIGMS T32-

GM008181 (to LMR), NIH/NINDS T32-NS063391 (to LMR). JBA, LMR, MP, and AK declare no financial conflicts of interest.

\section{ORCID}

Jason Bondoc Alipio: https://orcid.org/0000-0001-9315-345X

Lace Marie Riggs: https://orcid.org/0000-0003-4368-4089

Asaf Keller: https://orcid.org/0000-0001-8727-663X 


\section{References}

Abbas A-K, Villers A, Ris L (2015) Temporal phases of long-term potentiation (LTP): myth or fact? Rev Neuroscience 26:507-546.

Ahmadalipour A, Ghodrati-Jaldbakhan S, Samaei SA, Rashidy-Pour A (2018) Deleterious effects of prenatal exposure to morphine on the spatial learning and hippocampal BDNF and long-term potentiation in juvenile rats: Beneficial influences of postnatal treadmill exercise and enriched environment. Neurobiol Learn Mem 147:54-64.

Alipio JB, Brockett AT, Fox ME, Tennyson SS, deBettencourt CA, El $\square$ Metwally D, Francis NA, Kanold PO, Lobo MK, Roesch MR, Keller A (2021a) Enduring consequences of perinatal fentanyl exposure in mice. Addict Biol 26:e12895.

Alipio JB, Haga C, Fox ME, Arakawa K, Balaji R, Cramer N, Lobo MK, Keller A (2021b) Perinatal fentanyl exposure leads to long-lasting impairments in somatosensory circuit function and behavior. J Neurosci 41:3400-3417.

Ayres AJ (1964) Tactile functions. Their relation to hyperactive and perceptual motor behavior. Am J Occup Ther Official Publ Am Occup Ther Assoc 18:6-11.

Baltaci SB, Mogulkoc R, Baltaci AK (2019) Molecular Mechanisms of Early and Late LTP. Neurochem Res 44:281-296.

Baroncelli L, Braschi C, Spolidoro M, Begenisic T, Sale A, Maffei L (2010) Nurturing brain plasticity: impact of environmental enrichment. Cell Death Differ 17:1092-1103.

Bayat M, Sharifi MD, Haghani M, Shabani M (2015) Enriched environment improves synaptic plasticity and cognitive deficiency in chronic cerebral hypoperfused rats. Brain Res Bull 119:34-40.

Botanas CJ, Lee H, Peña JB de la, Peña IJ dela, Woo T, Kim HJ, Han DH, Kim B-N, Cheong JH (2016) Rearing in an enriched environment attenuated hyperactivity and inattention in the Spontaneously Hypertensive Rats, an animal model of Attention-Deficit Hyperactivity Disorder. Physiol Behav 155:30-37.

Brecht M (2017) The Body Model Theory of Somatosensory Cortex. Neuron 94:985-992.

Brenes JC, Padilla M, Fornaguera J (2009) A detailed analysis of open-field habituation and behavioral and neurochemical antidepressant-like effects in postweaning enriched rats. Behav Brain Res 197:125-137.

Byrnes EM, Vassoler FM (2018) Modeling prenatal opioid exposure in animals: Current findings and future directions. Front Neuroendocrin 51:1-13.

Cabelli RJ, Allendoerfer KL, Radeke MJ, Welcher AA, Feinstein SC, Shatz CJ (1996) Changing Patterns of Expression and Subcellular Localization of TrkB in the Developing Visual System. J Neurosci 16:7965-7980.

Chaplan SR, Bach FW, Pogrel JW, Chung JM, Yaksh TL (1994) Quantitative assessment of tactile allodynia in the rat paw. J Neurosci Meth 53:55-63.

Chen X, Li Y, Kline AE, Dixon CE, Zafonte RD, Wagner AK (2005) Gender and environmental effects on regional brain-derived neurotrophic factor expression after experimental traumatic brain injury. Neuroscience 135:11-17. 
Clancy B, Darlington RB, Finlay BL (2001) Translating developmental time across mammalian species. Neuroscience 105:7-17.

Clem RL, Celikel T, Barth AL (2008) Ongoing in Vivo Experience Triggers Synaptic Metaplasticity in the Neocortex. Science 319:101-104.

Cubas MM de, Field T (1993) Children of methadone-dependent women. Am J Orthopsychiat 63:266-276.

Degenhardt L et al. (2018) The global burden of disease attributable to alcohol and drug use in 195 countries and territories, 1990-2016: A systematic analysis for the Global Burden of Disease Study 2016. Lancet Psychiatry 5:987-1012.

Deuis JR, Dvorakova LS, Vetter I (2017) Methods used to evaluate pain behaviors in rodents. Front Mol Neurosci 10:284.

Dixon WJ (1965) The up-and-down method for small samples. J Am Stat Assoc 60:967.

Dow-Edwards D, Iijima M, Stephenson S, Jackson A, Weedon J (2014) The effects of prenatal cocaine, post-weaning housing and sex on conditioned place preference in adolescent rats. Psychopharmacology 231:1543-1555.

Eckert MJ, Bilkey DK, Abraham WC (2010) Altered plasticity in hippocampal CA1, but not dentate gyrus, following long-term environmental enrichment. J Neurophysiol 103:3320 3329.

Editorial (2018) Recommendations for the design and analysis of in vivo electrophysiology studies. J Neurosci 38:5837-5839.

Feng R, Rampon C, Tang YP, Shrom D, Jin J, Kyin M, Sopher B, Miller MW, Ware CB, Martin GM, Kim SH, Langdon RB, Sisodia SS, Tsien JZ (2001) Deficient neurogenesis in forebrainspecific presenilin-1 knockout mice is associated with reduced clearance of hippocampal memory traces. Neuron 32:911-926.

Foster TC, Gagne J, Massicotte G (1996) Mechanism of altered synaptic strength due to experience: relation to long-term potentiation. Brain Res 736:243-250.

Gaulke LJ, Horner PJ, Fink AJ, McNamara CL, Hicks RR (2005) Environmental enrichment increases progenitor cell survival in the dentate gyrus following lateral fluid percussion injury. Mol Brain Res 141:138-150.

Haight SC, Ko JY, Tong VT, Bohm MK, Callaghan WM (2018) Opioid use disorder documented at delivery hospitalization — United States, 1999-2014. Morbidity Mortal Wkly Rep 67:845-849.

Hanes AL, Koesters AG, Fong M, Altimimi HF, Stellwagen D, Wenner P, Engisch KL (2020) Divergent synaptic scaling of miniature EPSCs following activity blockade in dissociated neuronal cultures. J Neurosci 40:4090-4102.

Irvine GI, Logan B, Eckert M, Abraham WC (2006) Enriched environment exposure regulates excitability, synaptic transmission, and LTP in the dentate gyrus of freely moving rats. Hippocampus 16:149-160. 
Jannetto PJ, Helander A, Garg U, Janis GC, Goldberger B, Ketha H (2019) The fentanyl epidemic and evolution of fentanyl analogs in the United States and the European Union. Clin Chem 65:242-253.

Johnson NL, Carini L, Schenk ME, Stewart M, Byrnes EM (2011) Adolescent Opiate Exposure in the Female Rat Induces Subtle Alterations in Maternal Care and Transgenerational Effects on Play Behavior. Frontiers Psychiatry 2:29.

Kavalali ET (2015) The mechanisms and functions of spontaneous neurotransmitter release. Nat Rev Neurosci 16:5-16.

Kilkenny C, Browne W, Cuthill IC, Emerson M, Altman DG, Group NRGW (2010) Animal research: Reporting in vivo experiments: The ARRIVE guidelines. Brit J Pharmacol 160:1577-1579.

Kim J, Alger BE (2001) Random response fluctuations lead to spurious paired-pulse facilitation. J Neurosci 21:9608-9618.

Kivistö K, Nevalainen P, Lauronen L, Tupola S, Pihko E, Kivitie-Kallio S (2014) Somatosensory and auditory processing in opioid-exposed newborns with neonatal abstinence syndrome: a magnetoencephalographic approach. J Maternal-fetal Neonatal Medicine 28:2015-2019.

Korkhin A, Zubedat S, Aga-Mizrachi S, Avital A (2020) Developmental effects of environmental enrichment on selective and auditory sustained attention.

Psychoneuroendocrino 111:104479.

Landis SC et al. (2012) A call for transparent reporting to optimize the predictive value of preclinical research. Nature 490:187-191.

Mégevand P, Troncoso E, Quairiaux C, Muller D, Michel CM, Kiss JZ (2009) Long-term plasticity in mouse sensorimotor circuits after rhythmic whisker stimulation. J Neurosci 29:5326-5335.

Monday HR, Younts TJ, Castillo PE (2018) Long-Term Plasticity of Neurotransmitter Release: Emerging Mechanisms and Contributions to Brain Function and Disease. Annu Rev Neurosci 41:1-24.

Montarolo F, Martire S, Perga S, Spadaro M, Brescia I, Allegra S, Francia SD, Bertolotto A (2019) NURR1 deficiency is associated to ADHD-like phenotypes in mice. Transl Psychiat 9:207.

Motanis H, Seay MJ, Buonomano DV (2018) Short-Term Synaptic Plasticity as a Mechanism for Sensory Timing. Trends Neurosci 41:701-711.

Mychasiuk R, Muhammad A, Kolb B (2014) Environmental enrichment alters structural plasticity of the adolescent brain but does not remediate the effects of prenatal nicotine exposure. Synapse 68:293-305.

Nicoll RA (2017) A Brief History of Long-Term Potentiation. Neuron 93:281-290.

Nithianantharajah J, Hannan AJ (2006) Enriched environments, experience-dependent plasticity and disorders of the nervous system. Nat Rev Neurosci 7:697-709. 
Niu L, Cao B, Zhu H, Mei B, Wang M, Yang Y, Zhou Y (2009) Impaired in vivo synaptic plasticity in dentate gyrus and spatial memory in juvenile rats induced by prenatal morphine exposure. Hippocampus 19:649-657.

Nygaard E, Slinning K, Moe V, Walhovd KB (2016) Behavior and attention problems in eightyear-old children with prenatal opiate and poly-substance exposure: A longitudinal study. Plos One 11:e0158054.

O’Donnell JK, Halpin J, Mattson CL, Goldberger BA, Gladden RM (2017) Deaths involving fentanyl, fentanyl analogs, and U-47700 — 10 States, July-December 2016. Mmwr Morbidity Mortal Wkly Rep 66:1197-1202.

Ornoy A, Segal J, Bar $\square$ Hamburger R, Greenbaum C (2001) Developmental outcome of school $\square$ age children born to mothers with heroin dependency: Importance of environmental factors. Dev Medicine Child Neurology 43:668-675.

Passineau MJ, Green EJ, Dietrich WD (2001) Therapeutic effects of environmental enrichment on cognitive function and tissue integrity following severe traumatic brain injury in rats. Exp Neurol 168:373-384.

Ramirez DM, Kavalali ET (2011) Differential regulation of spontaneous and evoked neurotransmitter release at central synapses. Curr Opin Neurobiol 21:275-282.

Rijzingen IMS van, Gispen WH, Spruijt BM (1997) Postoperative environmental enrichment attenuates fimbria-fornix lesion-induced impairments in Morris maze performance. Neurobiol Learn Mem 67:21-28.

Rioult-Pedotti M-S, Friedman D, Donoghue JP (2000) Learning-Induced LTP in Neocortex. Science 290:533-536.

Robertson CE, Baron-Cohen S (2017) Sensory perception in autism. Nat Rev Neurosci 18:671684.

Ryan CL, Pappas BA (1990) Prenatal exposure to antiadrenergic antihypertensive drugs: Effects on neurobehavioral development and the behavioral consequences of enriched rearing. Neurotoxicol Teratol 12:359-366.

Schlagal CR, Dunn TJ, Xu P, Felsing DE, Merritt CR, Manja S, Fox RG, Buffington SA, Saade G, Dineley KT, Yu Y, Cunningham KA, Wu P (2021) Maternal opioid exposure culminates in perturbed murine neurodevelopment and hyperactive phenotype in adolescence. Neuroscience 463:272-287.

Schulz PE, Fitzgibbons JC (1997) Differing Mechanisms of Expression for Short- and LongTerm Potentiation. J Neurophysiol 78:321-334.

Schwartz AN, Reyes LM, Meschke LL, Kintziger KW (2021) Prenatal opioid exposure and ADHD childhood symptoms: A meta-analysis. Children 8:106.

Sherman LJ, Ali MM, Mutter R, Larson J (2019) Mental Disorders Among Children Born With Neonatal Abstinence Syndrome. Psychiatr Serv 70:151-151.

Smail MA, Smith BL, Nawreen N, Herman JP (2020) Differential impact of stress and environmental enrichment on corticolimbic circuits. Pharmacol Biochem Be 197:172993. 
Sutter MB, Leeman L, Hsi A (2014) Neonatal opioid withdrawal syndrome. Obstet Gyn Clin N Am 41:317-334.

Taniguchi N, Takada N, Kimura F, Tsumoto T (2000) Actions of brain $\square$ derived neurotrophic factor on evoked and spontaneous EPSCs dissociate with maturation of neurones cultured from rat visual cortex. J Physiology 527:579-592.

Ting JT, Daigle TL, Chen Q, Feng G (2014) Patch-clamp methods and protocols. Methods Mol Biology 1183:221-242.

Toyoda H, Zhao M-G, Ulzhöfer B, Wu L-J, Xu H, Seeburg PH, Sprengel R, Kuner R, Zhuo M (2009) Roles of the AMPA receptor subunit GluA1 but not GluA2 in synaptic potentiation and activation of ERK in the anterior cingulate cortex. Mol Pain 5:1744-8069-5-46.

VanHouten JP, Rudd RA, Ballesteros MF, Mack KA (2019) Drug overdose deaths among women aged 30-64 years — United States, 1999-2017. Morbidity Mortal Wkly Rep 68:1-5.

Wade CL, Krumenacher P, Kitto KF, Peterson CD, Wilcox GL, Fairbanks CA (2013) Effect of chronic pain on fentanyl self-administration in mice. Plos One 8:e79239.

Wade CL, Schuster DJ, Domingo KM, Kitto KF, Fairbanks CA (2008) Supraspinallyadministered agmatine attenuates the development of oral fentanyl self-administration. Eur $\mathbf{J}$ Pharmacol 587:135-140.

Wang H, Xu X, Xu X, Gao J, Zhang T (2020) Enriched environment and social isolation affect cognition ability via altering excitatory and inhibitory synaptic density in mice hippocampus. Neurochem Res 45:2417-2432.

Wei F, Li W, Ma B, Deng X, Zhang L, Zhao L, Zheng T, Jing Y (2021) Experiences affect social behaviors via altering neuronal morphology and oxytocin system. Psychoneuroendocrino 129:105247.

Whiteman VE, Salemi JL, Mogos MF, Cain MA, Aliyu MH, Salihu HM (2014) Maternal opioid drug use during pregnancy and its impact on perinatal morbidity, mortality, and the costs of medical care in the United States. J Pregnancy 2014:1-8.

Wille-Bille A, Bellia F, García AMJ, Miranda-Morales RS, D’Addario C, Pautassi RM (2020)

Early exposure to environmental enrichment modulates the effects of prenatal ethanol exposure upon opioid gene expression and adolescent ethanol intake. Neuropharmacology 165:107917.

Winkelman TNA, Villapiano N, Kozhimannil KB, Davis MM, Patrick SW (2018) Incidence and costs of neonatal abstinence syndrome among infants with Medicaid: 2004-2014. Pediatrics 141:e20173520.

Wu X-S, Xue L, Mohan R, Paradiso K, Gillis KD, Wu L-G (2007) The origin of quantal size variation: Vesicular glutamate concentration plays a significant role. J Neurosci 27:30463056.

Wu Y, Mitra R (2020) Prefrontal-hippocampus plasticity reinstated by an enriched environment during stress. Neurosci Res.

Yazdanfar N, Farnam A, Sadigh-Eteghad S, Mahmoudi J, Sarkaki A (2021) Enriched environment and social isolation differentially modulate addiction-related behaviors in male 
offspring of morphine-addicted dams: The possible role of $\mu$-opioid receptors and $\Delta$ FosB in the brain reward pathway. Brain Res Bull 170:98-105.

Zhao M-G, Toyoda H, Lee Y-S, Wu L-J, Ko SW, Zhang X-H, Jia Y, Shum F, Xu H, Li B-M, Kaang B-K, Zhuo M (2005) Roles of NMDA NR2B subtype receptor in prefrontal long-term potentiation and contextual fear memory. Neuron 47:859-872.

Zheng J-J, Li S-J, Zhang X-D, Miao W-Y, Zhang D, Yao H, Yu X (2014) Oxytocin mediates early experience-dependent cross-modal plasticity in the sensory cortices. Nat Neurosci 17:391-399. 